\title{
A search for Dark Matter in the centre of the Earth with the IceCube neutrino detector.
}

\author{
The IceCube Collaboration ${ }^{\dagger}$ \\ ${ }^{\dagger}$ http://icecube.wisc.edu/collaboration/authors/icrc15_icecube \\ E-mail: Jan.Kunnen@vub.ac.be
}

\begin{abstract}
Many models predict new particles that have the properties of a Weakly Interacting Massive Particle (WIMP) and could explain the dark matter observed in the universe. Heavy celestial bodies, such as the Earth, could capture these WIMPs and accumulate them. Over time the WIMPs will self-annihilate and may produce standard model particles, including neutrinos. Large scale neutrino telescopes, such as the cubic kilometre IceCube Neutrino Observatory located at the South Pole, can be used to search for such neutrino fluxes.

The dark matter annihilation rate in the centre of the Earth, and thus the resulting neutrino flux depend on the local Dark Matter density and the mass of the Dark Matter particle. This flux could be within reach of a large neutrino detector like IceCube. We present the results of the first search for Earth WIMPs with the IceCube detector.
\end{abstract}

Corresponding authors: J. Kunnen ${ }^{* 1}$, J. Lünemann ${ }^{1}$,

${ }^{1}$ Interuniversity Institute for High Energies, Vrije Universiteit Brussel, Belgium

The 34th International Cosmic Ray Conference,

30 July- 6 August, 2015

The Hague, The Netherlands

${ }^{*}$ Speaker. 


\section{Introduction}

Some of the most promising dark matter candidates are Weakly Interacting Massive Particles (WIMPs). In the Minimally Supersymmetric Standard Model (MSSM), the WIMP can take the form of the lightest neutralino. Dark matter particles from the galactic halo might become bound in the gravitational potential of the solar system as it passes through the galaxy. These particles may then scatter weakly on nuclei in the Earth and lose energy, becoming trapped by the Earth. Over time, this leads to an accumulation of dark matter in the centre of the Earth. The accumulated dark matter may then self annihilate at a rate that is proportional to its density, generating a flux of neutrinos which is spectrally dependent on the annihilation channel and neutralino mass.

Expected neutrino event rates and energies depend on the specific model and distribution of dark matter under consideration and the chemical composition of the Earth. Taking these variables into consideration leads to a neutrino-induced muon flux from the centre of the Earth varying between $10^{-8}-10^{5}$ per $\mathrm{km}^{2}$ per year for WIMPs with masses in the $\mathrm{GeV}-\mathrm{TeV}$ range [1]. AMANDA [2] and Super-K [3] already ruled out muon fluxes above $\sim 10^{3}$ per $\mathrm{km}^{2}$ per year. The possibility of looking for even lower fluxes due to the increased size of the IceCube neutrino observatory with respect to previous detectors, motivates the continuation of searches for neutrinos coming from WIMP annihilations in the centre of the Earth.

\section{The IceCube Neutrino Telescope}

IceCube is located in the glacial ice at the geographic South Pole. It consists of an array of digital optical modules (DOMs), designed to collect the Cherenkov radiation produced by high energy, neutrino-induced charged leptons travelling through the detector volume. By recording the number of Cherenkov photons and their arrival times, the direction and energy of the charged lepton, and consequently that of the parent neutrino, can be reconstructed.

IceCube is an approximately $1 \mathrm{~km}^{3}$ instrumented volume consisting of 86 strings, each containing 60 DOMs, deployed between $1450 \mathrm{~m}$ and $2450 \mathrm{~m}$ depth in the ice [4]. Of these 86,8 strings at the centre of IceCube comprise DeepCore, a more densely instrumented sub-array equipped with high quantum efficiency DOMs.

While the large ice overburden provides a shield against downward going, cosmic ray induced muons with energies $\lesssim 500 \mathrm{GeV}$ at the surface, most analyses also use the Earth as a filter and focus on upward going neutrinos. Additionally, low energy analyses mainly focus on DeepCore as a fiducial volume and use the surrounding IceCube strings as an active veto to reduce penetrating muon backgrounds. The search for WIMP annihilation signatures at the center of the Earth takes advantage of these two background rejection techniques as the expected signal will be vertically upgoing and of low energy.

\section{Dark Matter from the centre of the Earth}

WIMPs accumulated in the centre of the Earth will produce a unique signature in IceCube as vertically upgoing muons. The number of detected neutrino-induced muons depends on the neutralino annihilation rate $\Gamma_{A}$. If the capture rate $C$ is constant in time $t, \Gamma_{A}$ is given by [1] 


$$
\Gamma_{A}=\frac{C}{2} \tanh ^{2}\left(\frac{t}{\tau}\right), \tau=\left(C C_{A}\right)^{-1 / 2} .
$$

The equilibrium time $\tau$ is defined as the time when the annihilation rate and the capture rate are equal, where $C_{A}$ is a constant depending on the WIMP number density. For the Earth, this equilibrium time is of the order of $10^{11}$ years if the spin-independent WIMP-proton cross section is $\sigma_{p}^{S I} \sim 10^{-43} \mathrm{~cm}^{2}$ [5]. The age of the solar system is $t_{\circ} \approx 4.5 \mathrm{Gyr}$ and so $t_{\circ} / \tau \ll 1$, we thus expect that $\Gamma_{A} \propto C^{2}$, i.e. the higher the capture rate, the higher the annihilation rate and thus the muon flux.

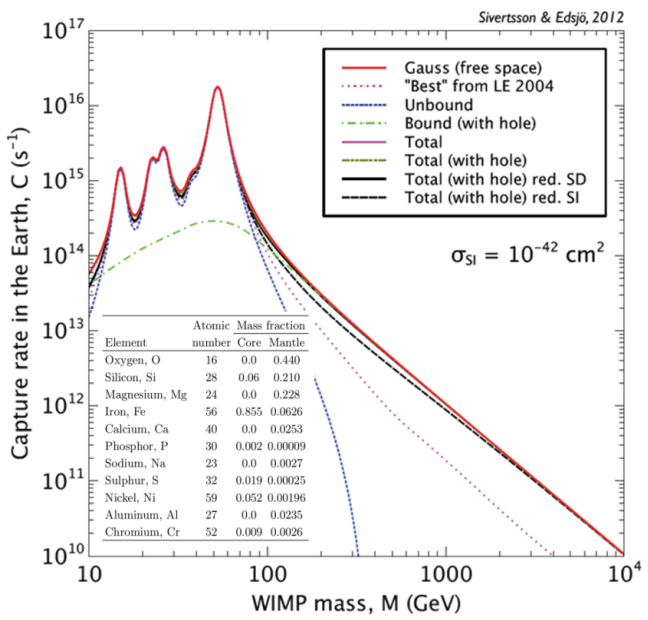

Figure 1: This figure shows the rate at which dark matter particles are captured to the interior of the Earth, for a scattering cross section of $\sigma=$ $10^{-42} \mathrm{~cm}^{2}$. The peaks correspond to resonant capture on the most abundant elements (listed in the table, abundances are given in weight \%) considered in the Earth model [6], ${ }^{16} \mathrm{O},{ }^{24} \mathrm{Mg}$, ${ }^{28} \mathrm{Si}$ and ${ }^{56} \mathrm{Fe}$ and their isotopes. A dark matter halo density of $\rho_{\mathrm{X}}=0.3 \mathrm{GeV} \mathrm{cm}^{-3}$ is assumed [7].

The rate at which WIMPs are captured in the Earth depends on the mass (which is unknown), the local WIMP density (which can be measured) and the velocity of the WIMPs (which cannot be measured observationally, and one has to resort to simulations to try to estimate it). If the WIMP mass is nearly identical to that of one of the nuclear species in the Earth, the capture rate will increase considerably, as is shown in Fig. 1. It should be noted that recent direct detection limits [8] exclude cross sections larger than $\sigma_{p}^{S I}=10^{-43} \mathrm{~cm}^{2}$ over a wide range of WIMP masses. This implies that the normalization in Fig. 1 should be corrected downward by about an order of magnitude lower, as the cross section that is assumed in the calculation for the capture rate is $\sigma=10^{-42} \mathrm{~cm}^{2}$ [7], but it does not affect the overall shape.

The capture rate could be higher if the WIMPs have a low velocity with respect to the Earth due to the small escape velocity, $v_{e s c}$, of WIMPs inside the Earth. This escape velocity varies from $11 \mathrm{~km} / \mathrm{s}$ at the mantle to $15 \mathrm{~km} / \mathrm{s}$ at the center of the Earth. WIMPs with high velocities will thus only be captured at the center, whereas low velocity WIMPs may be captured anywhere inside the Earth. As different models for the halo lead to different WIMP velocity distributions, these distributions are very sensitive to theoretical assumptions. The most popular halo model is the Standard Halo Model (SHM), which is a smooth, spherically symmetric density component with a non-rotating Gaussian velocity distribution [9]. Galaxy formation simulations including baryons indicate, however, that there is at least one local macrostructural component beyond the SHM. Some of these simulations show that a thick disc of dark matter, with kinematics similar to the thick disc of stars and a mid-plane density of $0.25-1.5$ times the local dark halo density $[10,11]$, is formed as the baryonic disc of the Milky Way draws satellites closer to the disc plane by dynamical 
friction, where they are disrupted by tides [12]. The particles in this dark disc have a lower relative velocity to the Earth compared to the SHM. Therefore, less scattering is needed for the particles in the dark disc to become gravitationally captured. This dark disk would lead to a higher capture rate and therefore a higher neutrino-induced muon flux at detectors such as IceCube.

\section{Analysis}

The last published Earth WIMP search was performed with the neutrino data recorded by the AMANDA detector over the three year period 1997 - 99 [2]. No excess above the expected atmospheric neutrino background was found in this search and therefore an upper limit was set on the WIMP annihilation rate $\Gamma_{A}$, in the Earth (dotted line in Fig. 6)

This analysis uses the first year of IC86 data, which has been taken between May 2011 and May 2012. In order to do everything in a blind way, the event selection is optimized by using simulations to estimate the background and signal events. The background simulation consists of atmospheric muons that are simulated with CORSIKA [13] and atmospheric neutrinos that are simulated with GENIE [14] for neutrinos below $190 \mathrm{GeV}$ and NuGeN [15] above. The signal simulations that are used in the analysis are performed using WimpSim [16]. WimpSim is a code that describes the capture and annihilation of WIMPs inside the Sun or the Earth, collects all neutrinos that emerge and lets these propagate out of the Sun/Earth to the detector. The code includes neutrino interactions and neutrino oscillations in a fully consistent three-flavour treatment.

To be sensitive to a wide range of WIMP masses, the analysis is split in two parts, one that is optimised for low masses and the other for higher masses.
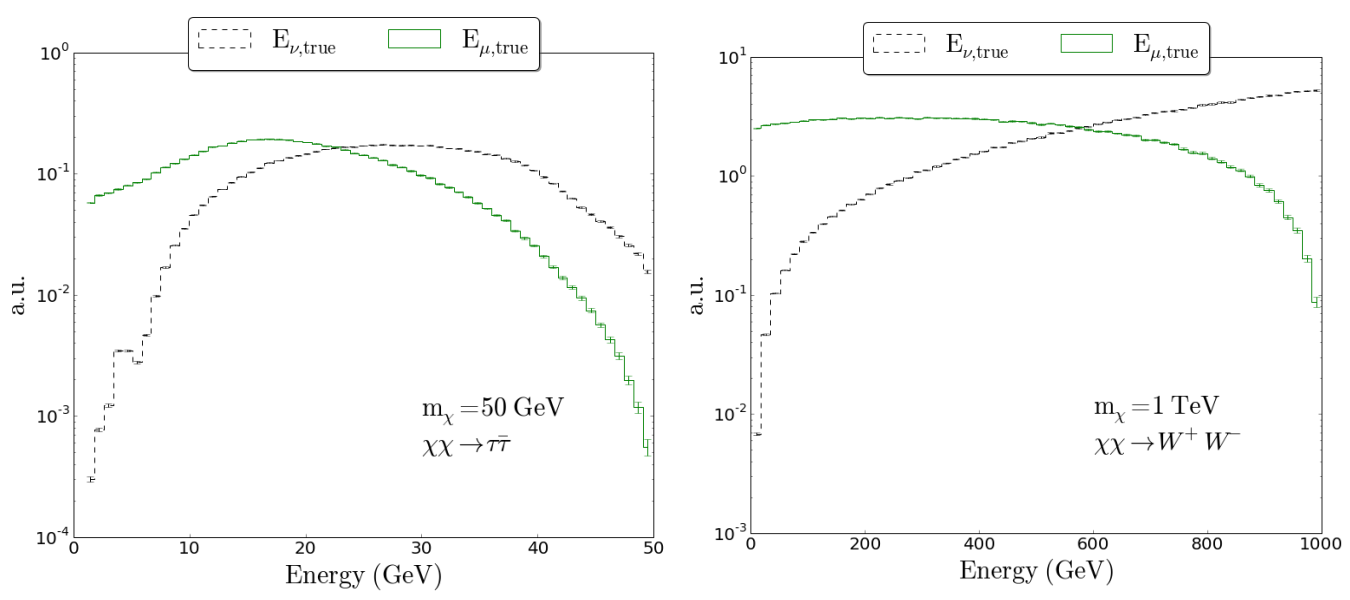

Figure 2: The normalized energy distributions for signal neutrinos and signal neutrino-induced muons, for a WIMP mass of $50 \mathrm{GeV}$ (left) and $1 \mathrm{TeV}$ (right).

The low mass analysis is optimised on WIMPs with a mass $m_{\chi}=50 \mathrm{GeV}$, that annihilate into $\tau \bar{\tau}$. As the capture rate is highest for WIMPs for this mass (see Fig. 1), the annihilation and thus neutrino rate are also highest. The high mass optimisation is done on WIMPs with a mass $m_{\chi}=1 \mathrm{TeV}$, that annihilate into $W^{+} W^{-}$. The expected neutrino spectrum (solid lines) and the neutrino-induced muon spectrum (dashed lines) for $50 \mathrm{GeV}$ WIMPs annihilating into $\tau \bar{\tau}$ and 
$1 \mathrm{TeV}$ WIMPs annihilating into $W^{+} W^{-}$are shown in Fig. 2. Note that the expected muon energy for the $50 \mathrm{GeV}$ WIMPs is lower than $50 \mathrm{GeV}$, so DeepCore detector will be crucial in this part of the analysis.

The neutrino-induced muon rate in IceCube, coming from WIMPs in the centre of the Earth can not be higher than $\sim 10^{3}$ muons per year $[2,3]$, which is a very low rate, compared to the $2.5 \mathrm{kHz}$ rate at which IceCube is taking data. The data are dominated by atmospheric muons $(\mathrm{kHz}$ rate), which can be removed via selection cuts, as explained below. These cuts lower the data rate by $\sim 6$ orders of magnitude, to get to the level where the data are mainly consisting of atmospheric neutrino events ( $\mathrm{mHz}$ rate). As atmospheric neutrino events with the same direction and energy as signal neutrino events are irreducible, a statistical analysis is performed on this neutrino sample, to look for an excess from the centre of the Earth.

The first level of cuts are done on the whole sample, i.e. before splitting the data into a low and a high energy sample. This is done to reduce the data rate to $\sim 1 \mathrm{~Hz}$, such that more precise (and more time-consuming) reconstructions can be used to calculate the energy on which the splitting will be based. These first cuts consist of a selection of online filters that tag upgoing events, followed by linear cuts on direction and interaction vertex of the lepton. These variables are not correlated with the energy of the neutrino and have thus similar efficiencies for different WIMP masses.

The variables that are used for cuts at this level are the reconstructed zenith angle, the reconstructed interaction vertex and the average drift of hits in the vertical $(z)$ direction. The zenith angle cut can not be too strict, as we aim to retain a signal free control region in which the agreement between data and background simulation will be tested. This is necassary as the detector acceptance is zenith dependent and it is thus not possible to define an off-source region in this analysis. Therefore we need to rely on simulations to understand the background, requiring a detailed understanding of systematic uncertainties. Based on these considerations, the zenith angle cut is chosen such that all events with a reconstructed zenith angle $<120^{\circ}$ are removed, and the agreement between data and background simulation can be tested in the region $<150^{\circ}$. The other cut values are chosen by looping over all possible combinations of cut values and checking which combination brings down the background to the $\mathrm{Hz}$ level, while removing as little signal as possible.

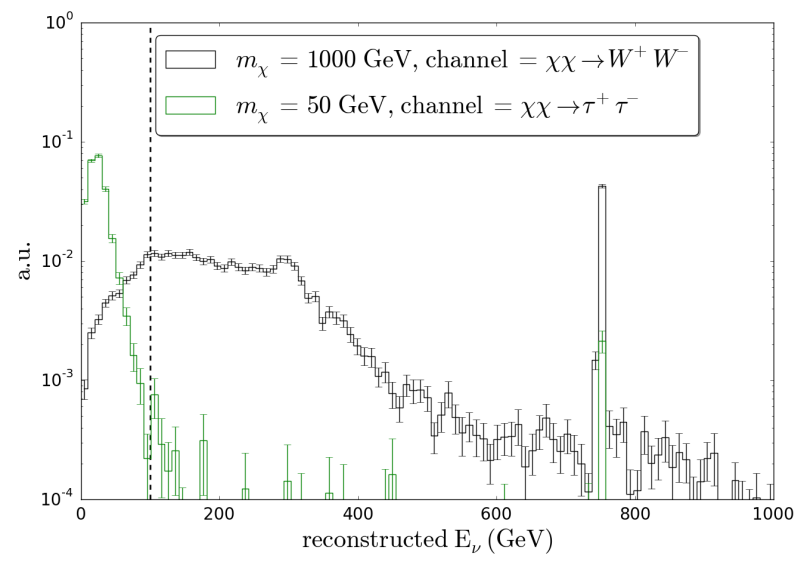

Figure 3: The reconstructed energy distributions for $50 \mathrm{GeV}$ and $1 \mathrm{TeV}$ Earth WIMPs. The vertical dashed line shows where the data sets are split. The peak at $\sim 750 \mathrm{GeV}$ comes from tracks with a reconstructed length of $2 \mathrm{~km}$, which is the value that is set by the algorithm when the length of the muon cannot be reconstructed (e.g. when it is not contained in the detector). As these events are generally bright events, it is a good feature that they end up in the high energy sample.

After this first cut level, the data rate goes down to $\sim 3 \mathrm{~Hz}$, while $30 \%-60 \%$ of the signal 
(depending on WIMP mass and channel) is kept. The data is still dominated by atmospheric muons at this level. Now that the data is at this low rate, it can be reprocessed using more precise (and more time-consuming) reconstructions.

One of these reconstructions is an energy reconstruction that is used in oscillation analyses in IceCube [17]. The reconstructed energies for $50 \mathrm{GeV}$ and $1 \mathrm{TeV}$ WIMP neutrinos are shown in Fig. 3. A division at $100 \mathrm{GeV}$, shown as a vertical line in this figure, is used to split the datasets into low and high energy samples which are statistically independent and will be optimized and analyzed separately.

Both analyses make use of Boosted Decision Trees (BDT), which is a machine learning technique that is designed to optimally separate signal from background [18] by assigning a score between -1 (background-like) and +1 (signal-like) to each event. Before training the BDT, one has to be sure that the simulation is representing the experimental data in a correct way, for this reason some more linear cuts are made prior to the BDT training in order to get good agreement between data and simulation. After this step, the experimental data rates are on the order of $100 \mathrm{mHz}$, and the data are still dominated by atmospheric muons. The BDTs are then trained on variables that are not too correlated, and that have good data-simulation agreement.

In the low energy optimization, the BDT training samples consist of simulated $50 \mathrm{GeV}$ WIMP neutrinos and experimental data for the signal and background set respectively.
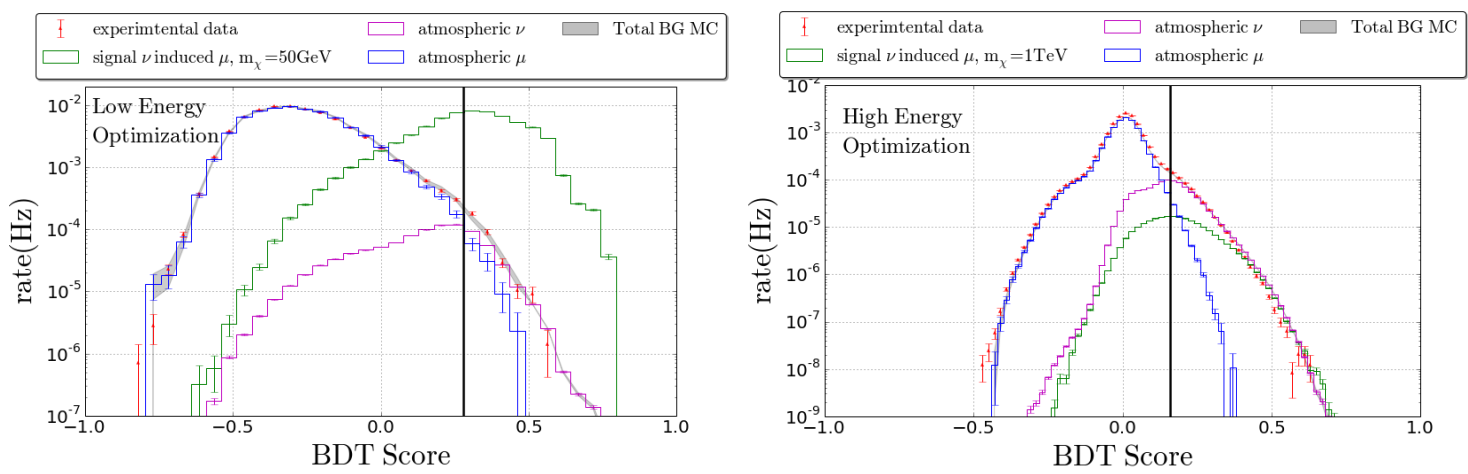

Figure 4: The plot shows the BDT score distributions the low energy analysis (left) and for the high energy analysis using the Pull-Validation method (right). Signal distributions are upscaled to be visible in the plot. Signal and backgrounds are compared to experimental data from 10\% of the first year of IC86 data. The vertical lines indicate the final cut value used in each analysis, where high scores to the right of the line are retained.

Because the opening angle between the neutrino and its daughter lepton is inversely proportional to the energy of the neutrino, WIMP neutrino-induced muons in the high energy analysis are going to be narrowly concentrated into vertical zenith angles, whereas in the low energy analysis they will be spread over a wider range of zenith angles. To train the BDT in the high energy analysis to find upgoing neutrino-induced candidates generally (rather than straight vertical events specifically), an generic muon neutrino simulation weighted to the energy spectrum of $1 \mathrm{TeV}$ WIMP neutrinos is used to simulate the signal.

The cuts on the BDT score are chosen such that the sensitivities of the analyses are optimal. The sensitivities are calculated with a likelihood ratio hypothesis test based on the values of the reconstructed zenith, using the Feldman-Cousins unified approach [19]. The required probability 
densities for signal and background are both calculated from simulations, as this analysis has no off-source region. The background sample that is left after the cut on BDT score mainly consists of atmospheric neutrinos and only has a small amount of atmospheric muon events. Smoothening methods are used to deal with this problem of small statistics.

The high energy analysis uses the Pull-Validation method [20], which is a method in which several BDTs (200 in the case of the present analysis) are trained on small subsets that are randomly resampled from the complete dataset. The variation of the BDT output between the trainings can be interpreted as a probability density function (PDF) for each event. This PDF can be used to calculate a weight that is applied to each event instead of making a binary cut decision. With this method not only the BDT score distribution is smoothened (Fig. 4-left), but also the distributions that are made after a cut on the BDT score. In particular the reconstructed zenith distribution used in the likelihood calculation is smooth, as events that would be removed when using a single BDT could now be kept, albeit with a smaller weight.

The low energy analysis tackles the problem of poor statistics of the atmospheric muon background sample in a different way. In this part of the analysis, only a single BDT is trained (Fig. 4right), and after the cut on the BDT score, the reconstructed zenith distribution is smoothened using Kernel Density Estimation (KDE) [21, 22], which is a method that smoothens each observed data point over a local neighbourhood of that data point.

The reconstructed zenith distributions after the cuts on the BDT scores are shown in Fig. 5. In these plots, the complete dataset of the first year of IC86 data is shown.

\section{Results and conclusions}
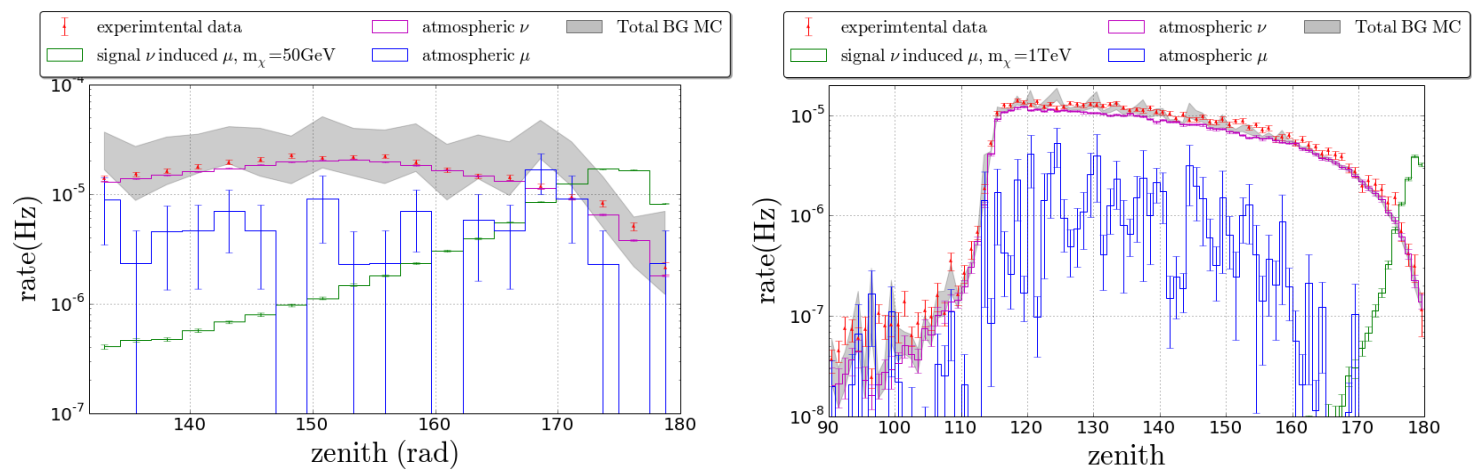

Figure 5: The plot shows the unblinded reconstructed zenith distributions of 1 year of IC86 data for the low energy analysis (left) and the high energy analysis using the Pull-Validation method (right). Signal distributions are upscaled to be visible in the plot. The gray areas indicate the background distributions with the 1 sigma uncertainties, including both statistical and systematic uncertainties for the left plot, and statistical uncertainties for the right plot.

As can be seen in Fig. 5, no statistically significant excess is found from the direction of the centre of the Earth, allowing us to set limits on the neutrino flux from the centre of the Earth in the $\mathrm{GeV}-\mathrm{TeV}$ range. These limits are calculated by using the final reconstructed zenith angle distributions as probability density functions in the likelihood calculation, using the Feldman-Cousins 
unified approach [19]. For the signal, the zenith distribution at each WIMP mass is calculated with WimpSim and for the background the smoothened zenith distributions of the remaining simulated atmospheric muons and neutrinos are used.

The limits on the WIMP annihilation rate in the Earth $\Gamma_{A}$ for 1 year of IC86 data (20112012) $)^{1}$ are shown in Fig. 6 and are compared with the AMANDA limits [2] which is the last Earth WIMP neutrino search. An improvement of a factor 10 has been found, meaning that a whole new part of the theoretical phase space can be excluded with this analysis.

\section{References}

[1] T. Bruch et al., Phys. Lett. B 675 (2009) 250 [astro-ph/ 0902.4001 ].

[2] A. Achterberg et al., Astropart. Phys. 26 (2006) 129 [astro-ph/0202370v3].

[3] S. Desai et al., Phys. Rev. D 70 (2004) 083523 [hep-ex/ 0404025$].$

[4] IceCube Collaboration, A. Achterberg, et al., Astropart. Phys. 26 (2006) 155 [astro-ph/0604450].

[5] G. Jungman et al., Phys. Rep. 267 (1996) 195 [hep-ph / 9506380$].$

[6] W.F. McDonough and S. Sun, Chemical Geology 120 (1995) 223.

[7] S. Sivertsson and J. Edsjö, Phys. Rev. D 85 (2012) 123514 [astro-ph/1201.1895].

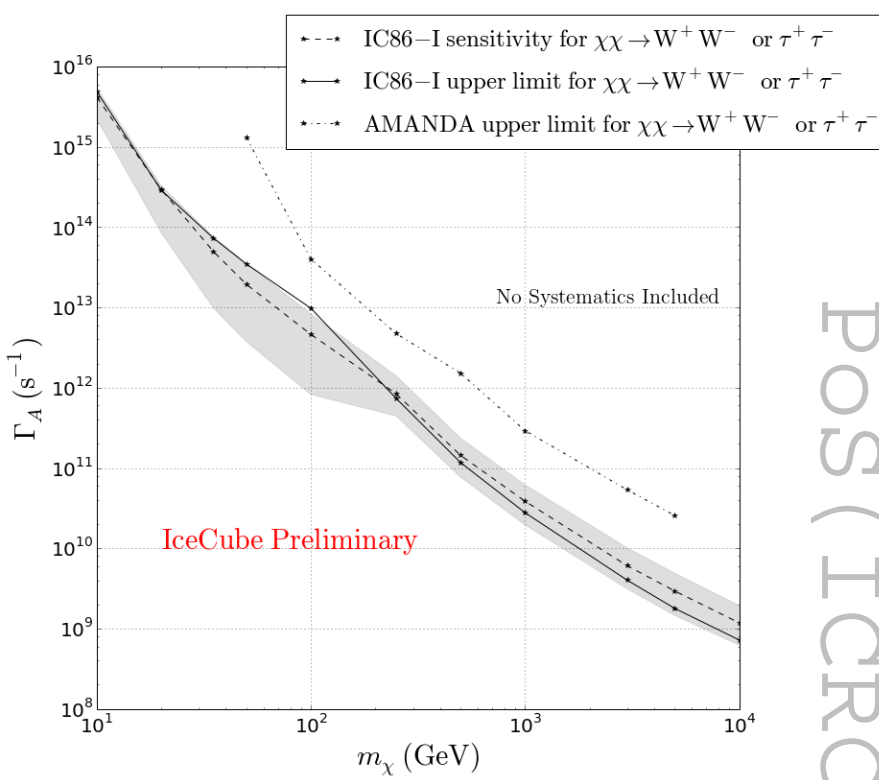

Figure 6: The upper limits (solid line) and sensitivities (dashed line) with 1 sigma uncertainty (gray band, not including systematics) on the annihilation rate in the Earth $\Gamma_{A}$ for 1 year of IC86 data as a function of the WIMP mass. The dotted line shows the latest upper limit on the annihilation rate, which was calculated with AMANDA data.

[8] E. Aprile et al., Phys. Rev. Lett. 109 (2012) 181301 [astro-ph/1207. 5988].

[9] K. Freese et al., Phys. Rev. D 37 (1988) 3388.

[10] J. Read et al., MNRAS 389 (2008) 1041 [astro-ph/ 0803.2714 ].

[11] J. Read et al., MNRAS 397 (2009) 44 [astro-ph/ 0902.0009 ].

[12] G. Lake, Astrophys. J. 98 (1989) 1554.

[13] D. Heck et al., FZKA Report 6019 (1998).

[14] C. Andreopoulos et al., Nucl. Instrum. Meth. A 614 (2010) 87 [astro-ph / 0905 . 2517].

[15] A. Gazizov et al., Comput. Phys. Commun. 172 (2005) 203 [a st ro-ph / 0406439 ].

[16] J. Edsjö, WimpSim Neutrino Monte Carlo, http://copsosx03.fysik.su.se/wimpsim/.

[17] IceCube Collaboration, M. G. Aartsen, et al., Phys. Rev. D 91 (2015) 072004 [hep-ex/1410.7227].

[18] A. Hoecker et al., PoS A CAT 040 (2007) [physics / 0703039 ].

[19] G. J. Feldman and R. D. Cousins, Phys. Rev. D 57 (1998) 3873 [physics / 9711021 ].

[20] IceCube Collaboration, $P O S(I C R C 2015) 1211$ these proceedings.

[21] M. Rosenblatt, The Annals of Mathematical Statistics 27 (1956) 832.

[22] E. Parzen, The Annals of Mathematical Statistics 33 (1962) 1065.

\footnotetext{
${ }^{1}$ These limits don't contain systematic uncertainties yet.
} 